\title{
RANDOM DYNAMICAL SYSTEMS WITH JUMPS AND WITH A FUNCTION TYPE INTENSITY
}

\author{
JOANNA KUBIENIEC
}

\begin{abstract}
In paper 4 there are considered random dynamical systems with randomly chosen jumps acting on Polish spaces. The intensity of this process is a constant $\lambda$. In this paper we formulate criteria for the existence of an invariant measure and asymptotic stability for these systems in the case when $\lambda$ is not constant but a Lipschitz function.
\end{abstract}

\section{Introduction}

In paper [4] there are considered a finite family of dynamical systems with randomly chosen jumps acting on a given Polish space. It is an example of a non-diffusion model and is similar to the so-called piecewise-deterministic Markov process introduced by Davis [1]. In paper [5] author study Markov process which is a solution of some stochastic differential equations driven by jump-type process. This process is defined with the help of only one dynamical system. In contrast to [4] the intensity of the process considered in [5] is not constant.

In this paper we apply the not constant process intensity to the finite family of random dynamical systems with jumps. We formulate criteria for stability and the existence of a stationary measure for these models.

It should be noted that there is a large range of application of dynamical systems in physics and biology like the short noise [8], the photo conductive

Received: 13.01.2016. Revised: 12.04.2016. Accepted: 19.04.2016.

(2010) Mathematics Subject Classification: 37A30, 60J75, 93D20.

Key words and phrases: dynamical systems, asymptotic stability, Markov operators. 
detectors [8], the growth of the size of structural populations [2], and many others.

The organization of the paper is as follows. Section 2 contains some notations and definitions from the theory of Markov operators. In Section 3 we formulate the problem to be considered. The existence of invariant measure and asymptotic stability of the considered dynamical system is included in Section 4. At the end of Section 4 we take under consideration some model, similar to the model of gene expression from [7] and we make some assumption under which we obtain asymptotic stability for this model.

\section{Preliminaries}

Let $(Y, \rho)$ be a Polish space. We denote by $B(x, r)$ the open ball with center at $x$ and radius $r$. For any set $A \subset Y, \operatorname{cl} A, \operatorname{diam}_{\rho}(A), \mathbb{1}_{A}$ stand for the closure, diameter, and indicator function of $A$, respectively.

We denote by $\mathrm{B}(Y)$ the $\sigma$-algebra of Borel subsets of $Y$, by $M=M(Y)$ the family of all finite Borel measures on $Y$, and by $M_{s}$ the space of all finite signed Borel measures on $Y$. We write $M_{1}=M_{1}(Y)$ for the family of all $\mu \in M$ such that $\mu(Y)=1$.

We denote by $B(Y)$ the space of all bounded Borel measurable functions $f: Y \rightarrow R$ and $C(Y)$ the subspace of all continuous functions. Both spaces are considered with the supremum norm $\|\cdot\|_{0}$. For $f \in B(Y)$ and $\mu \in M_{s}$ we write

$$
\langle f, \mu\rangle=\int_{Y} f(x) \mu(d x) .
$$

We introduce in $M_{s}$ the Fortet-Mourier norm $\|\cdot\|_{\rho}$ given by

$$
\|\mu\|_{\rho}=\sup \left\{|\langle f, \mu\rangle|: f \in F_{\rho}\right\} \quad \text { for } \mu \in M_{s},
$$

where $F_{\rho}$ is the set of all $f \in C(Y)$ such that $|f(x)| \leq 1$ and $|f(x)-f(y)| \leq$ $\rho(x, y)$ for $x, y \in Y$.

We say that a sequence $\left\{\mu_{n}\right\}_{n \geq 1}, \mu_{n} \in M$, converges weakly to a measure $\mu \in M$ if

$$
\lim _{n \rightarrow \infty}\left\langle f, \mu_{n}\right\rangle=\langle f, \mu\rangle \quad \text { for every } f \in C(Y) .
$$

We introduce the class $\Phi$ of functions $\varphi: R_{+} \rightarrow R_{+}$satisfying the following conditions: 
(i) $\varphi$ is continuous and $\varphi(0)=0$,

(ii) $\varphi$ is nondecreasing and concave, i.e.

$$
\sum_{k=1}^{n} \alpha_{k} \varphi\left(y_{k}\right) \leq \varphi\left(\sum_{k=1}^{n} \alpha_{k} y_{k}\right), \quad \text { where } \alpha_{k} \geq 0, \sum_{k=1}^{n} \alpha_{k}=1
$$

(iii) $\varphi(x)>0$ for $x>0$ and $\lim _{x \rightarrow \infty} \varphi(x)=\infty$.

We denote by $\Phi_{0}$ the family of all functions satisfying (i) and (ii). A necessary and sufficient condition for a concave function $\varphi$ to be subadditive on $(0, \infty)$ is that $\varphi(0+) \geq 0$. From this result we immediately obtain the triangle inequality for $\rho_{\varphi}=\varphi \circ \rho$. Thus for every $\varphi \in \Phi$ the function $\rho_{\varphi}$ is a metric on $Y$. We write $F_{\varphi}$ and $\|\cdot\|_{\varphi}$ instead of $F_{\rho_{\varphi}}$ and $\|\cdot\|_{\rho_{\varphi}}$, respectively. The proof of the following result can be found in [6].

Proposition 2.1. Let a function $\omega \in \Phi_{0}$ satisfies the Dini condition

$$
\int_{0}^{\epsilon} \frac{\omega(t)}{t} d t<\infty \quad \text { for some } \epsilon>0 \text {. }
$$

Let $a \in[0,1)$. Then inequality

$$
\omega(t)+\varphi(a t) \leq \varphi(t) \quad \text { for } t \geq 0
$$

admits a solution in $\Phi$.

We say that a vector $\left(p_{1}, \ldots, p_{N}\right)$, where $p_{i}: Y \rightarrow[0,1]$, is a probability vector if

$$
\sum_{i=1}^{N} p_{i}(x)=1 \quad \text { for } x \in Y
$$

A matrix $\left[p_{i j}\right]_{i, j}$, where $p_{i j}: Y \rightarrow[0,1]$ for $i, j \in\{1, \ldots, N\}$, is a probability matrix if

$$
\sum_{j=1}^{N} p_{i j}(x)=1 \quad \text { for } x \in Y \text { and } i \in\{1, \ldots, N\} \text {. }
$$

An operator $P: M \rightarrow M$ is called a Markov operator if

$$
P\left(\lambda_{1} \mu_{1}+\lambda_{2} \mu_{2}\right)=\lambda_{1} P \mu_{1}+\lambda_{2} P \mu_{2} \quad \text { for } \lambda_{1}, \lambda_{2} \in R_{+} \text {and } \mu_{1}, \mu_{2} \in M
$$

and

$$
P \mu(Y)=\mu(Y) \quad \text { for } \mu \in M \text {. }
$$


The Markov operator can be extended to the space $M_{s}$. A linear operator $U: B(Y) \rightarrow B(Y)$ is called dual to $P$ if

$$
\langle U f, \mu\rangle=\langle f, P \mu\rangle \quad \text { for } f \in B(Y) \text { and } \mu \in M .
$$

A Markov operator $P$ is called a Markov-Feller operator if it has a dual operator $U$ such that

$$
U f \in C(Y) \quad \text { for } f \in C(Y) .
$$

An operator $P: M \rightarrow M$ is called nonexpansive if

$$
\left\|P \mu_{1}-P \mu_{2}\right\|_{\rho} \leq\left\|\mu_{1}-\mu_{2}\right\|_{\rho} \quad \text { for } \mu_{1}, \mu_{2} \in M_{1} .
$$

A measure $\mu$ is called invariant (or stationary) with respect to $P$ if $P \mu=\mu$. A Markov operator $P$ is called asymptotically stable if there exists a stationary measure $\mu_{*} \in M_{1}$ such that

$$
\lim _{n \rightarrow \infty}\left\|P^{n} \mu-\mu_{*}\right\|_{\rho}=0 \quad \text { for every } \mu \in M_{1} .
$$

Obviously a measure $\mu_{*}$ satisfying the above condition is unique.

We say that a metric $\bar{\rho}$ is equivalent to metric $\rho$ if the classes of bounded sets and convergent sequences in the spaces $(Y, \bar{\rho})$ and $(Y, \rho)$ coincide. If $(Y, \rho)$ is Polish space and $\bar{\rho}, \rho$ are equivalent, then the space $(Y, \bar{\rho})$ is still a Polish space.

An operator $P: M \rightarrow M$ is called essentially nonexpansive if there exists a metric $\bar{\rho}$ equivalent to $\rho$ such that $P$ is nonexpansive with respect to the norm $\|\cdot\|_{\bar{\rho}}$.

We denote by $C_{\epsilon}(Y), \epsilon>0$, the family of all closed sets $C$ for which there exists a finite set $\left\{z_{1}, z_{2}, \ldots, z_{n}\right\} \subset Y$ such that $C \subset \bigcup_{i=1}^{n} B\left(z_{1}, \epsilon\right)$. An operator $P$ is called semi-concentrating if for every $\epsilon>0$ there exist $C \in C_{\epsilon}(Y)$ and $\theta>0$ such that

$$
\liminf _{n \rightarrow \infty} P^{n} \mu(C)>\theta \quad \text { for } \mu \in M_{1} .
$$

For $\mu \in M_{1}$ we consider the limit set

$$
L(\mu)=\left\{\nu \in M_{1} \text { : there exists }\left\{n_{k}\right\} \subset\{n\} \text { such that } \lim _{k \rightarrow \infty}\left\|P^{n_{k}} \mu-\nu\right\|_{\rho}=0\right\}
$$

and

$$
L\left(M_{1}\right)=\bigcup_{\mu \in M_{1}} L(\mu)
$$


Let $A \in \mathrm{B}(Y)$. We say that a measure $\mu \in M$ is concentrated on $A$ if $\mu(Y \backslash A)=0$. We denote by $M_{1}^{A}$ the set of all probability measures concentrated on $A$.

An operator $P$ is called globally concentrating if for every $\epsilon>0$ and every bounded Borel set $A \in \mathrm{B}(Y)$ there exist a bounded Borel set $B \in \mathrm{B}(Y)$ and $n_{0} \in N$ such that

$$
P^{n} \mu(B) \geq 1-\epsilon \quad \text { for } n \geq n_{0}, \mu \in M_{1}^{A} .
$$

Define

$$
E(P)=\left\{\epsilon>0: \inf _{\mu \in M_{1}} \liminf _{n \rightarrow \infty} P^{n} \mu(A)>0 \text { for some } A \in C_{\epsilon}(Y)\right\} .
$$

REMARK 2.2. If a Markov operator $P$ is globally concentrating, then $E(P) \neq \emptyset$.

Remark 2.3. If $\inf E(P)=0$, then $P$ is semi-concentrating.

A continuous function $V: Y \rightarrow[0, \infty)$ is called Lyapunov function if

$$
\lim _{\rho\left(x, z_{0}\right) \rightarrow \infty} V(x)=\infty
$$

for some $z_{0} \in Y$.

\section{Formulation of the problem}

Let $(Y, \rho)$ be a separable Banach space, $R_{+}=[0, \infty)$ and $I=\{1, \ldots, N\}$, $\Theta$ be a compact metric space. Let $S_{i}: R_{+} \times Y \rightarrow Y, i \in I$, be a finite sequence of semidynamical systems, i.e.

$$
S_{i}(0, x)=x \quad \text { for } i \in I, x \in Y
$$

and

$$
S_{i}(s+t, x)=S_{i}\left(s, S_{i}(t, x)\right) \quad \text { for } s, t \in R_{+}, i \in I \text { and } x \in Y \text {. }
$$


We are given probability vector $\left(p_{1}, \ldots, p_{n}\right)$, where $p_{i}: Y \rightarrow[0,1], i, j \in I$, a matrix of probabilities $\left[p_{i j}\right]_{i, j \in I}, p_{i j}: Y \rightarrow[0,1]$ and continuous functions $q: Y \times \Theta \rightarrow Y, \lambda: Y \rightarrow(0, \infty)$, such that

$$
\underline{\lambda}=\inf _{x \in Y} \lambda(x)>0 .
$$

Let $\left(\Omega, \Sigma\right.$, prob) be a probability space and $\left\{t_{n}\right\}_{n \geq 0}$ be an increasing sequence of random variables $t_{n}: \Omega \rightarrow R_{+}$with $t_{0}=0$. Let $\left\{\eta_{n}\right\}_{n \in N}$ be a sequence of independent identically distributed random elements with values in a compact metric space $\Theta$, their distribution will be denoted by $\kappa$.

We consider the space $X=Y \times I$ endowed with the metric $\bar{\rho}$ given by

$$
\bar{\rho}((x, i),(y, j))=\rho(x, y)+\rho_{c}(i, j) \quad \text { for } x, y \in Y, i, j \in I,
$$

where

$$
\rho_{c}(i, j)= \begin{cases}c & \text { if } i \neq j, \\ 0 & \text { if } i=j,\end{cases}
$$

and the constant $c$ will be chosen later on.

We define a new sequence of semidynamical systems

$$
\bar{S}_{k}: R_{+} \times X \rightarrow X \quad \text { for } k=1,2 \ldots
$$

by

$$
\bar{S}_{k}(t,(x, s))= \begin{cases}(x, s) & \text { if } t=0 \\ \left(S_{k}(t, x), k\right) & \text { if } t>0\end{cases}
$$

We define

$$
\bar{q}\left(\bar{S}_{k}(t,(x, s)), \eta\right):=q\left(S_{k}(t, x), \eta\right)
$$

for $k=1,2, \ldots, t \in R_{+}, x \in Y, s \in I$, and $\eta \in \Theta$.

The action of randomly chosen dynamical systems, with randomly chosen jumps, at random moments $t_{k}$ can be roughly described as follows. We choose an initial point $x_{0} \in Y$ and randomly select a transformation $S_{k}$ from the set $\left\{S_{1}, \ldots, S_{N}\right\}$ in such a way that the probability of choosing $S_{k}$ is equal to $p_{k}\left(x_{0}\right)$, i.e., we define $\xi_{0}: \Omega \rightarrow I$ such that $\operatorname{prob}\left(\xi_{0}=k \mid x_{0}=x\right)=p_{k}(x)$. We obtain pair $\left(x_{0}, k\right)$. We define

$$
x_{1}=q\left(S_{k}\left(t_{1}, x_{0}\right), \eta_{1}\right),
$$

where $\operatorname{prob}\left(t_{1} \leq t \mid \xi_{0}=k\right.$ and $\left.x_{0}=x\right)=1-e^{-\int_{0}^{t} \lambda\left(S_{k}(s, x)\right) d s}$. 
We randomly choose an integer $i_{1} \in\{1, \ldots, N\}$ with probability $p_{k i_{1}}\left(x_{1}\right)$, i.e., we define $\xi_{1}: \Omega \rightarrow I$ such that $\operatorname{prob}\left(\xi_{1}=i_{1} \mid x_{1}=x\right.$ and $\left.\xi_{0}=k\right)=p_{k i_{1}}(x)$. We obtain $\left(x_{1}, i_{1}\right)$ and define

$$
x_{2}=q\left(S_{i_{1}}\left(t_{2}-t_{1}, x_{1}\right), \eta_{2}\right)=\bar{q}\left(\bar{S}_{i_{1}}\left(t_{2}-t_{1},\left(x_{1}, k\right)\right), \eta_{2}\right)
$$

where $\operatorname{prob}\left(t_{2}-t_{1} \leq t \mid \xi_{1}=i_{1}\right.$ and $\left.x_{1}=x\right)=1-e^{-\int_{0}^{t} \lambda\left(S_{i_{1}}(s, x)\right) d s}$. We select $i_{2} \in\{1, \ldots, N\}$ with probability $p_{i_{1} i_{2}}\left(x_{2}\right)$, i.e., we define $\xi_{2}: \Omega \rightarrow I$, such that $\operatorname{prob}\left(\xi_{2}=i_{2} \mid x_{2}=x\right.$ and $\left.\xi_{1}=i_{1}\right)=p_{i_{1} i_{2}}(x)$. We obtain pair $\left(x_{2}, i_{2}\right)$. Finally, given $\left(x_{n}, i_{n}\right), n \geq 2$, we define

$$
x_{n+1}=q\left(S_{i_{n}}\left(t_{n+1}-t_{n}, x_{n}\right), \eta_{n+1}\right)=\bar{q}\left(\bar{S}_{i_{n}}\left(t_{n+1}-t_{n},\left(x_{n}, i_{n}\right)\right), \eta_{n+1}\right)
$$

where $\operatorname{prob}\left(t_{n+1}-t_{n} \leq t \mid \xi_{n}=i_{n}\right.$ and $\left.x_{n}=x\right)=1-e^{-\int_{0}^{t} \lambda\left(S_{i_{n}}(s, x)\right) d s}$. We select $i_{n+1} \in\{1, \ldots, N\}$ with probability $p_{i_{n} i_{n+1}}\left(x_{n+1}\right)$, i.e., we define $\xi_{n+1}: \Omega \rightarrow I$ such that $\operatorname{prob}\left(\xi_{n+1}=i_{n+1} \mid x_{n+1}=x\right.$ and $\left.\xi_{n}=i_{n}\right)=p_{i_{n} i_{n+1}}(x)$. We obtain $\left(x_{n+1}, i_{n+1}\right)$.

Above considerations we can describe using congruent sequences of random variables. Let $\left\{\xi_{n}\right\}_{n \geq 0}$ be sequence of random variables $\xi_{n}: \Omega \rightarrow I$. Let

$$
\begin{gathered}
\operatorname{prob}\left(\xi_{0}=i \mid x_{0}=x\right)=p_{i}(x), \\
\operatorname{prob}\left(\xi_{n}=s \mid x_{n}=x \text { and } \xi_{n-1}=k\right)=p_{k s}(x),
\end{gathered}
$$

for $n \geq 1, x, y \in Y, k, i \in I$. We assume that $\left\{\xi_{n}\right\}_{n \geq 0}$ and $\left\{\eta_{n}\right\}_{n \geq 0}$ independent of $\left\{t_{n}\right\}_{n \geq 0}$ and that for every $n \in N$ the variables $\eta_{1}, \ldots, \eta_{n-1}, \xi_{1}, \ldots, \xi_{n-1}$ are also independent. Given initial random variable $x_{0}$ and $\xi_{0}$, the sequence of the random variables $\left\{x_{n}\right\}_{n \geq 1}, x_{n}: \Omega \rightarrow Y$, is given by

$$
x_{n+1}=q\left(S_{\xi_{n}}\left(t_{n+1}-t_{n}, x_{n}\right), \eta_{n+1}\right) \quad \text { for } n=0,1 \ldots
$$

where

$$
\operatorname{prob}\left(t_{n+1}-t_{n} \leq t \mid \xi_{n}=s \text { and } x_{n}=x\right)=1-e^{-\int_{0}^{t} \lambda\left(S_{s}(z, x)\right) d z} .
$$

The integral is well defined, because $\underline{\lambda}>0$.

We will study the stochastic discrete process $\left\{\left(x_{n}, \xi_{n}\right)\right\}_{n \geq 0}$ (see [3, 4]). The evolution of the distributions $\mu_{n}$ on $X$ defined by

$$
\left.\mu_{n}(A)=\operatorname{prob}\left\{\left(x_{n}, \xi_{n}\right) \in A\right)\right\}
$$

for $A \in B_{X}$ can be described by an operator $P: M(X) \rightarrow M(X)$ such that

$$
\mu_{n+1}=P \mu_{n}
$$


where

$$
\begin{array}{r}
P \mu(A)=\sum_{s=1}^{N} \int_{X} \int_{0}^{\infty} \int_{\Theta} 1_{A}\left(q\left(S_{s}(t, x), \theta\right), s\right) e^{-L(t, x, k)} \\
\times \lambda\left(S_{k}(t, x)\right) p_{k s}(x) d \kappa(\theta) d t d \mu(x, k)
\end{array}
$$

and its dual operator $U: B(X) \rightarrow B(X)$ by

$$
\begin{aligned}
U f(x, k)=\sum_{s=1}^{N} \int_{0}^{\infty} \int_{\Theta} f\left(q\left(S_{s}(t, x), \theta\right), s\right) & e^{-L(t, x, k)} \\
& \times \lambda\left(S_{k}(t, x)\right) p_{k s}(x) d \kappa(\theta) d t
\end{aligned}
$$

where $L(t, x, k)=\int_{0}^{t} \lambda\left(S_{k}(z, x)\right) d z$.

\section{Invariant measure and asymptotic stability of $P$}

Let $(Y,\|\cdot\|)$ be a separable Banach space. In order to get the existence of invariant measure or asymptotic results for operator $P$ described by (3.1), we will need the following assumption:

(a) The transformations $S_{i}: \mathbb{R}_{+} \times Y \rightarrow Y, i \in I$ are continuous and there exists $x_{*} \in Y$ such that

$$
\begin{gathered}
\int_{0}^{\infty}\left\|S_{s}\left(t, x_{*}\right)-x_{*}\right\| e^{-\underline{\lambda} t} p_{k s}(x) d t<\infty \\
\int_{\Theta}\left\|q\left(x_{*}, \theta\right)\right\| d \kappa(\theta)<\infty .
\end{gathered}
$$

(b) The functions $p_{k s}: Y \rightarrow[0,1], k, s \in I$ satisfy the following conditions

$$
\begin{aligned}
\sum_{s=1}^{N}\left|p_{k s}(x)-p_{k s}(y)\right| & \leq \psi_{1}(\|x-y\|) \quad \text { for } x, y \in Y \text { and } k \in I, \\
\sigma & =\inf \left\{p_{k s}(x)\right\}>0
\end{aligned}
$$

where $\psi_{1} \in \Phi_{0}$ satisfy the Dini condition 2.1 . 
(c) We assume that there are constants $L \geq 1, \alpha \in R$ and $l_{q}>0, l_{\lambda}>0$ such that

$$
\sum_{s=1}^{N} p_{k s}(y)\left\|S_{s}(t, x)-S_{s}(t, y)\right\| \leq L e^{\alpha t}\|x-y\| \quad \text { for } x, y \in Y,
$$

$$
\|q(x, \cdot)-q(y, \cdot)\|_{L_{1}(\kappa)} \leq l_{q}\|x-y\| \quad \text { for } x, y \in Y,
$$

and

$$
\begin{aligned}
& |\lambda(x)-\lambda(y)| \leq l_{\lambda}\|x-y\| \quad \text { for } x, y \in Y, \\
& \underline{\lambda}=\inf _{x \in Y} \lambda(x)>0, \quad \bar{\lambda}=\sup _{x \in Y} \lambda(x)<\infty .
\end{aligned}
$$

The proofs of presented theorems are based on techniques shown in [4].

Theorem 4.1. Assume that the conditions (4.3)-4.6) are satisfied. If

$$
L l_{q} \bar{\lambda}+\alpha<\underline{\lambda},
$$

then the operator $P$ defined by (3.1) is essentially nonexpansive.

Proof. Let $\psi_{1} \in \Phi_{0}$ be given by condition (4.3). Define $\bar{\psi}: R_{+} \rightarrow R$ by

$$
\bar{\psi}(t)=\psi_{2}(t)+b t
$$

for some $b>0$, where $\psi_{2}=\frac{\bar{\lambda}}{\lambda} \psi_{1}$. It is easy to see that $\bar{\psi} \in \Phi_{0}$ and satisfies the hypotheses of Proposition 2.1. There exists $\varphi \in \Phi$ such that

$$
\bar{\psi}(t)+\varphi(a t) \leq \varphi(t), \quad \text { where } a=\frac{L l_{q} \bar{\lambda}}{\underline{\lambda}-\alpha}<1 .
$$

Since $\varphi \in \Phi$ we may choose $c \in \mathbb{R}_{+}$such that $\varphi(c)>2$. Consider the metric $\hat{\rho}$

$$
\hat{\rho}((x, i),(y, i))=\|x-y\|+\rho_{c}(i, j) \quad \text { for } x, y \in Y, i, j \in I .
$$

Let $f \in F_{\varphi}$. It is evident that $U f \in C(X)$, where $U$ is given by equation (3.2) and

$$
|U f(x, k)| \leq \sum_{s=1}^{N} \int_{0}^{\infty} \int_{\Theta}\left|f\left(q\left(S_{s}(t, x), \theta\right), s\right) e^{-L(t, x, k)} \lambda\left(S_{k}(t, x)\right) p_{k s}(x)\right| d \kappa(\theta) d t .
$$


Hence

$$
\begin{aligned}
|U f(x, k)| & \leq \sum_{s=1}^{N} \int_{0}^{\infty} e^{-L(t, x, k)} \lambda\left(S_{k}(t, x)\right) d t p_{k s}(x) \\
& =-\sum_{s=1}^{N} \int_{0}^{\infty} \frac{d}{d t} e^{-L(t, x, k)} d t p_{k s}(x)=1
\end{aligned}
$$

To complete the proof it is enough to show that

$$
|U f(x, k)-U f(y, s)| \leq \varphi(\hat{\rho}((x, k),(x, s)))
$$

for $(x, i),(x, j) \in X$. Since $\rho_{c}(k, s)=c$ for $k \neq s, \varphi(c)>2$, and $|f|<1$, the above condition is satisfied for $k \neq s$. For $k=s$ we obtain

$$
\begin{aligned}
\mid U f(x, k)- & U f(y, k) \mid \\
\leq & \sum_{s=1} \int_{0}^{\infty} \int_{\Theta} \mid f\left(q\left(S_{s}(t, x), \theta\right), s\right) e^{-L(t, x, k)} \lambda\left(S_{k}(t, x)\right) p_{k s}(x) \\
& -f\left(q\left(S_{s}(t, y), \theta\right), s\right) e^{-L(t, y, k)} \lambda\left(S_{k}(t, y)\right) p_{k s}(y) \mid d \kappa(\theta) d t \\
\leq & I_{1}+I_{2}
\end{aligned}
$$

where

$$
\begin{aligned}
I_{1}=\sum_{s=1}^{N} \int_{0}^{\infty} \int_{\Theta} \mid f\left(q\left(S_{s}(t, x), \theta\right), s\right)- & f\left(q\left(S_{s}(t, y), \theta\right), s\right) \mid \\
& \times e^{-L(t, y, k)} \lambda\left(S_{k}(t, y)\right) p_{k s}(y) d \kappa(\theta) d t
\end{aligned}
$$

and

$$
\begin{aligned}
I_{2}= & \sum_{s=1}^{N} \int_{0}^{\infty} \int_{\Theta}\left|f\left(q\left(S_{s}(t, x), \theta\right), s\right)\right| \\
& \quad \times\left|e^{-L(t, x, k)} \lambda\left(S_{k}(t, x)\right) p_{k s}(x)-e^{-L(t, y, k)} \lambda\left(S_{k}(t, y)\right) p_{k s}(y)\right| d \kappa(\theta) d t .
\end{aligned}
$$


Since $f \in F_{\varphi}$

$$
\begin{aligned}
& I_{1} \leq \sum_{s=1} \int_{0}^{\infty} \int_{\Theta} \varphi\left(\left\|q\left(S_{s}(t, x), \theta\right)-q\left(S_{s}(t, y), \theta\right)\right\|\right) \\
& \quad \times e^{-L(t, y, k)} \lambda\left(S_{k}(t, y)\right) p_{k s}(y) d \kappa(\theta) d t \\
& \leq \varphi\left(\int_{0}^{\infty} l_{q} L \bar{\lambda} e^{\alpha t}\|x-y\| e^{-\underline{\lambda} t} d t\right)=\varphi\left(\frac{L l_{q} \bar{\lambda}}{\bar{\lambda}-\alpha}\|x-y\|\right) .
\end{aligned}
$$

We now calculate $I_{2}$. We have

$$
\begin{aligned}
I_{2} \leq & \sum_{s=1}^{N} \int_{0}^{\infty}\left|e^{-L(t, x, k)} \lambda\left(S_{k}(t, x)\right) p_{k s}(x)-e^{-L(t, y, k)} \lambda\left(S_{k}(t, y)\right) p_{k s}(y)\right| d t \\
\leq & \sum_{s=1}^{N} \int_{0}^{\infty} e^{-L(t, x, k)} p_{k s}(x)\left|\lambda\left(S_{k}(t, x)\right)-\lambda\left(S_{k}(t, y)\right)\right| d t \\
& +\sum_{s=1}^{N} \int_{0}^{\infty} \lambda\left(S_{k}(t, y)\right)\left|e^{-L(t, x, k)} p_{k s}(x)-e^{-L(t, y, k)} p_{k s}(y)\right| d t .
\end{aligned}
$$

From the mean-value theorem we obtain that for every fixed $t \in[0, \infty), x, y \in$ $Y$, and $k=1, \ldots, N$

$$
\left|e^{-L(t, x, k)}-e^{-L(t, y, k)}\right|=e^{-L_{1}}|L(t, x, k)-L(t, y, k)|,
$$

where $L(t, x, k)<L_{1}(t, x, y, k)<L(t, y, k)$. Since $\underline{\lambda}>0$, for every $t \in[0, \infty)$, $x \in Y, k=1, \ldots, N$ we have $\underline{\lambda} t \leq L(t, x, k)$. We obtain

$$
\left|e^{-L(t, x, k)}-e^{-L(t, y, k)}\right| \leq e^{-\underline{\lambda} t}|L(t, x, k)-L(t, y, k)|
$$

for every $t \in[0, \infty), x, y \in Y$, and $k=1, \ldots, N$.

We go back to calculate $I_{2}$. We have

$$
\begin{aligned}
I_{2} \leq & l_{\lambda} \int_{0}^{\infty} e^{-\underline{\lambda} t}\left\|S_{k}(t, x)-S_{k}(t, y)\right\| d t \\
& +\sum_{s=1}^{N} \int_{0}^{\infty} \lambda\left(S_{k}(t, y)\right) e^{-L(t, y, k)}\left|p_{k s}(x)-p_{k s}(y)\right| d t \\
& +\int_{0}^{\infty} \lambda\left(S_{k}(t, y)\right)\left|e^{-L(t, x, k)}-e^{-L(t, y, k)}\right| d t .
\end{aligned}
$$


Thus

$$
\begin{aligned}
I_{2} & \leq \frac{l_{\lambda} L}{\sigma(\underline{\lambda}-\alpha)}\|x-y\|+\psi_{2}(\|x-y\|)+\frac{\bar{\lambda} l_{\lambda} L}{\sigma \underline{\lambda}(\underline{\lambda}-\alpha)}\|x-y\| \\
& =\psi_{2}(\|x-y\|)+b\|x-y\|,
\end{aligned}
$$

where $b=\frac{l_{\lambda} L(\bar{\lambda}+\underline{\lambda})}{\sigma \underline{\lambda}(\underline{\lambda}-\alpha)}>0$. We obtain that

$$
I_{2} \leq \psi_{2}(\|x-y\|)+b\|x-y\| .
$$

From 4.9, 4.10, and 4.8 we obtain

$$
\begin{aligned}
|U f(x, k)-U f(y, k)| & \leq \varphi\left(\frac{L l_{q} \bar{\lambda}}{\underline{\lambda}-\alpha}\|x-y\|\right)+\bar{\psi}(\|x-y\|) \\
& \leq \varphi(\|x-y\|) .
\end{aligned}
$$

Theorem 4.2. Assume that conditions 4.1)-4.7) are satisfied. Then operator $P$ given by equation (3.1) is semi-concentrating.

Proof. We show that there exists $a, b \in \mathbb{R}_{+}, a<1$, such that

$$
U V(x, k) \leq a V(x, k)+b,
$$

where

$$
V(x, k)=\|x\|,
$$

for $(x, k) \in X$. We have

$$
\begin{aligned}
U V(x, k) \leq \sum_{s=1}^{N} \int_{0}^{\infty} \int_{\Theta}\left\|q\left(S_{s}(t, x), \theta\right)-q\left(S_{s}\left(t, x_{*}\right), \theta\right)\right\| \\
\times e^{-L(t, x, k)} \lambda\left(S_{k}(t, x)\right) p_{k s}(x) d \kappa(\theta) d t \\
+\sum_{s=1}^{N} \int_{0}^{\infty} \int_{\Theta}\left\|q\left(S_{s}\left(t, x_{*}\right), \theta\right)-q\left(x_{*}, \theta\right)\right\| e^{-L(t, x, k)} \lambda\left(S_{k}(t, x)\right) p_{k s}(x) d \kappa(\theta) d t \\
+\sum_{s=1}^{N} \int_{0}^{\infty} \int_{\Theta}\left\|q\left(x_{*}, \theta\right)\right\| e^{-L(t, x, k)} \lambda\left(S_{k}(t, x)\right) p_{k s}(x) d \kappa(\theta) d t .
\end{aligned}
$$


Thus

$$
\begin{aligned}
U V(x, k) \leq & \sum_{s=1}^{N} l_{q} \int_{0}^{\infty}\left\|S_{s}(t, x)-S_{s}\left(t, x_{*}\right)\right\| e^{-L(t, x, k)} \lambda\left(S_{k}(t, x)\right) p_{k s}(x) d t \\
& +\sum_{s=1}^{N} l_{q} \int_{0}^{\infty}\left\|S_{s}\left(t, x_{*}\right)-x_{*}\right\| e^{-L(t, x, k)} \lambda\left(S_{k}(t, x)\right) p_{k s}(x) d t \\
& +\sum_{s=1}^{N} \int_{0}^{\infty} \int_{\Theta}\left\|q\left(x_{*}, \theta\right)\right\| e^{-L(t, x, k)} \lambda\left(S_{k}(t, x)\right) p_{k s}(x) d \kappa(\theta) d t \\
\leq & \frac{L l_{q} \bar{\lambda}}{} \underline{\underline{\lambda}-\alpha}\left\|x-x_{*}\right\|+\sum_{s=1}^{N} l_{q} \bar{\lambda} \int_{0}^{\infty}\left\|S_{s}\left(t, x_{*}\right)-x_{*}\right\| e^{-\underline{\lambda} t} p_{k s}(x) d t \\
& +\frac{\bar{\lambda}}{\underline{\lambda}} \int_{\Theta}\left\|q\left(x_{*}, \theta\right)\right\| d \kappa(\theta) .
\end{aligned}
$$

We obtain

$$
U V(x, k) \leq \frac{L l_{q} \bar{\lambda}}{\underline{\lambda}-\alpha}\left\|x-x_{*}\right\|+b^{*} \leq a\|x\|+b
$$

where

$$
\begin{gathered}
a=\frac{L l_{q} \bar{\lambda}}{\underline{\lambda}-\alpha}, \quad b=b^{*}+a\|x\| \\
b^{*}=\sum_{s=1}^{N} l_{q} \bar{\lambda} \int_{0}^{\infty}\left\|S_{s}\left(t, x_{*}\right)-x_{*}\right\| e^{-\underline{\lambda} t} p_{k s}(x) d t+\frac{\bar{\lambda}}{\underline{\lambda}} \int_{\Theta}\left\|q\left(x_{*}, \theta\right)\right\| d \kappa(\theta) .
\end{gathered}
$$

From [9, Corollary 2.4.1] it follows that there exists a bounded set $A \in X$ such that

$$
\inf _{\mu \in M_{1}} \liminf _{n \rightarrow \infty} P^{n} \mu(A)>0
$$

which gives us that

$$
E(P)=\left\{\epsilon>0: \inf _{\mu \in M_{1}} \liminf _{n \rightarrow \infty} P^{n} \mu(A)>0 \text { for some } A \in C_{\epsilon}(X)\right\}
$$

is nonempty. We want to show that inf $E(P)=0$.

Suppose, contrary to our claim, that $\varepsilon^{*}=\inf E(P)>0$. We consider two cases: $\alpha<0$ and $\alpha \geq 0$, where $\alpha$ is given by condition 4.4 . 
CASE 1: $\alpha<0$. We may choose $z_{0} \in Y$ and $r>0$ such that

$$
\inf _{\mu \in M_{1}} \liminf _{n \rightarrow \infty} P^{n} \mu\left(B\left(z_{0}, r\right) \times I\right)>0 .
$$

Fix $t_{*}>0$ such that

$$
\varepsilon=4 r L l_{q} e^{\alpha t_{*}}<\varepsilon^{*}
$$

and set

$$
C_{\varepsilon}=\bigcup_{i \in I} \bigcup_{t \in\left[t_{*}, 2 t_{*}\right]} \bigcup_{\theta \in \Theta}\left(B\left(q\left(S_{i}\left(t, z_{0}\right), \theta\right), \varepsilon\right) \times I\right) .
$$

Observe that $C_{\varepsilon} \in C_{\varepsilon}(Y)$. From equation (3.1), for arbitrary $\mu \in M_{1}$ we have

$$
\begin{aligned}
P^{n+1} \mu\left(C_{\varepsilon}\right)=\sum_{s=1}^{N} \int_{X} \int_{0}^{\infty} \int_{\Theta} 1_{C_{\varepsilon}} & \left.\left(S_{s}(t, x), \theta\right), s\right) e^{-L(t, x, k)} \\
& \times \lambda\left(S_{k}(t, x)\right) p_{k s}(x) d \kappa(\theta) d t P^{n} \mu(d x, k) .
\end{aligned}
$$

For $x \in B\left(z_{0}, r\right)$ and $t>t_{*}$ we define

$$
\begin{aligned}
J(x, t) & =\left\{j \in I:\left\|S_{j}(t, x)-S_{j}\left(t, z_{0}\right)\right\| \leq 2 L e^{\alpha t}\left\|x-z_{0}\right\|\right\}, \\
\Theta_{0}(x, t) & =\left\{\theta \in \Theta:\left\|q\left(S_{j}(t, x), \theta\right)-q\left(S_{j}\left(t, z_{0}\right), \theta\right)\right\| \leq 2 l_{q}\left\|S_{j}(t, x)-S_{j}\left(t, z_{0}\right)\right\|\right\} .
\end{aligned}
$$

From assumption 4.5 it follows that $\kappa\left(\Theta_{0}(x, t)\right) \geq \frac{1}{2}$. Since

$$
\sum_{j \in I} p_{i j}(x)=1
$$

we have, by 4.4,

$$
\sum_{j \in J(x, t)} p_{i j}(x) \geq \frac{1}{2} \quad \text { for } i \in I .
$$

Let $x \in B\left(z_{0}, r\right), t \in\left[t_{*}, 2 t_{*}\right]$ and $\theta \in \Theta_{0}(x, t)$. Then for every $j \in J(x, t)$ we have

$$
\begin{aligned}
\left\|q\left(S_{j}(t, x), \theta\right)-q\left(S_{j}\left(t, z_{0}\right), \theta\right)\right\| & \leq 2 l_{q}\left\|S_{j}(t, x)-S_{j}\left(t, z_{0}\right)\right\| \\
& \leq l_{q} 4 L e^{\alpha t}\left\|x-z_{0}\right\|<\varepsilon .
\end{aligned}
$$


This gives $\left(q\left(S_{j}(t, x), \theta\right), j\right) \in C_{\varepsilon}$. Thus

$$
\begin{aligned}
& P^{n+1} \mu\left(C_{\varepsilon}\right) \\
& \geq \int_{B\left(z_{0}, r\right) \times I} \int_{t_{*}}^{2 t_{*}} \int_{\theta \in \Theta_{0}} \sum_{j \in J(x, t)} e^{-L(t, x, k)} \lambda\left(S_{k}(t, x)\right) p_{k j}(x) d t P^{n} \mu(d x, k) \\
& \geq \frac{1}{4} \underline{\lambda} e^{-\bar{\lambda} t_{*}}\left(1-e^{-\bar{\lambda} t_{*}}\right) P^{n} \mu\left(B\left(z_{0}, r\right) \times I\right) .
\end{aligned}
$$

From above we conclude

$$
\inf _{\mu \in M_{1}} \liminf _{n \rightarrow \infty} P^{n} \mu\left(C_{\varepsilon}\right)>0
$$

which contradicts the fact that $\varepsilon^{*}=\inf E(P)$. By Remark 2.3 , operator $P$ is semi-concentrating.

CASE 2: $\alpha \geq 0$. By 4.7 we have

$$
L l_{q}<\frac{\lambda}{\bar{\lambda}}-\frac{\alpha}{\bar{\lambda}}
$$

which implies that

$$
L l_{q}+\frac{\alpha}{\bar{\lambda}}<\frac{\lambda}{\bar{\lambda}} \leq 1
$$

We have $L l_{q}<1$. Choose $\eta, \delta, t_{*}>0$ such that

$$
(1+\eta)(1+\delta) L l_{q} e^{\alpha t_{*}}<1
$$

Finally, choose $\varepsilon_{0}>\varepsilon^{*}$ such that

$$
\varepsilon=(1+\eta)(1+\delta) L l_{q} e^{\alpha t_{*}} \varepsilon_{0}<\varepsilon^{*} .
$$

By the definition $E(P)$ there exists $A \in C_{\varepsilon_{0}}$ such that

$$
\beta=\inf _{\mu \in M_{1}} \liminf _{n \rightarrow \infty} P^{n} \mu(A)>0 .
$$

Without loss of generality we can assume that

$$
A=\bigcup_{k=1}^{m}\left(B\left(z_{k}, \varepsilon_{0}\right) \times I\right) .
$$


We now define

$$
C_{\varepsilon}=\bigcup_{j \in I} \bigcup_{t \in\left[0, t_{*}\right]} \bigcup_{\theta \in \Theta} \bigcup_{k=1}^{m}\left(B\left(q\left(S_{j}\left(t, z_{k}\right), \theta\right), \varepsilon\right) \times I\right) .
$$

Fix $\mu \in M_{1}$. It follows that there exists $k(n) \in\{1, \ldots, m\}$ such that

$$
P^{n} \mu\left(B\left(z_{k(n)}, \varepsilon_{0}\right) \times I\right) \geq \frac{\beta}{m} .
$$

For $x \in B\left(z_{k(n)}, \varepsilon_{0}\right)$ and $t<t_{*}$ we define

$$
\begin{aligned}
J(x, t) & =\left\{j \in I:\left\|S_{j}(t, x)-S_{j}\left(t, z_{k(n)}\right)\right\| \leq(1+\delta) L e^{\alpha t}\left\|x-z_{k(n)}\right\|\right\} \\
\Theta_{0}(x, t)=\left\{\theta \in \Theta: \| q\left(S_{j}(t, x), \theta\right)-\right. & q\left(S_{j}\left(t, z_{k(n)}\right), \theta\right) \| \\
& \left.\leq(1+\eta) l_{q}\left\|S_{j}(t, x)-S_{j}\left(t, z_{k(n)}\right)\right\|\right\} .
\end{aligned}
$$

From assumption 4.5$), \kappa\left(\Theta_{0}(x, t)\right) \geq \frac{\eta}{1+\eta}$. Similarly to the first case

$$
\sum_{j \in J(x, t)} p_{i j}(x) \geq \frac{\delta}{1+\delta} \quad \text { for } i \in I
$$

Fix $x \in B\left(z_{k(n)}, \varepsilon_{0}\right), t<t_{*}$ and $\theta \in \Theta_{0}$. We have

$$
\begin{aligned}
\left\|q\left(S_{j}(t, x), \theta\right)-q\left(S_{j}\left(t, z_{k(n)}\right), \theta\right)\right\| & \leq(1+\eta) l_{q} \| S_{j}(t, x)-S_{j}\left(t, z_{k(n)} \|\right. \\
& \leq l_{q}(1+\eta)(1+\delta) L e^{\alpha t}\left\|x-z_{k(n)}\right\| \\
& \leq l_{q}(1+\eta)(1+\delta) L e^{\alpha t_{*}} \varepsilon_{0}=\varepsilon .
\end{aligned}
$$

Thus $\left(q\left(S_{j}(t, x), \theta\right), j\right) \in C_{\varepsilon}$ and

$$
\begin{aligned}
P^{n+1} \mu\left(C_{\varepsilon}\right) \geq \sum_{j \in J(x, t)} \int_{B\left(z_{k(n)}, \varepsilon_{0}\right) \times I} \int_{0}^{t_{*}} \int_{\Theta_{0}(x, t)} e^{-L(t, x, k)} \lambda\left(S_{k}(t, x)\right) \\
\times p_{k j}(x) d \kappa(\theta) d t P^{n} \mu(d x, d k) \\
\geq \frac{\delta}{1+\delta} \frac{\eta}{1+\eta} \int_{0}^{t_{*}} e^{-\bar{\lambda} t} \underline{\lambda} P^{n}\left(B\left(z_{k(n)}, \varepsilon_{0}\right) \times I\right) \\
=\frac{\delta}{\bar{\lambda}(1+\delta)} \frac{\eta}{1+\eta}\left(1-e^{-\bar{\lambda} t_{*}}\right) P^{n}\left(B\left(z_{k(n)}, \varepsilon_{0}\right) \times I\right) .
\end{aligned}
$$


Combining this with 4.11 gives

$$
\liminf _{n \rightarrow \infty} P^{n} \mu\left(C_{\varepsilon}\right) \geq \frac{\eta \delta \beta}{m \bar{\lambda}(1+\eta)(1+\delta)}\left(1-e^{-\bar{\lambda} t_{*}}\right) P^{n}\left(B\left(z_{k(n)}, \varepsilon_{0}\right) \times I\right)>0
$$

but $\mu \in M_{1}$ was arbitrary and $\varepsilon<\varepsilon^{*}$, which is impossible. By Remark 2.3 we obtain that $P$ is semi-concentrating.

THEOREM 4.3. Assume that (4.1)-(4.7) are satisfied. Then operator $P$ defined by (3.1) has an invariant probability measure.

Proof. By Theorem 4.1 and 4.2 the operator $P$ is nonexpansive and semi-concentrating. Using [9, Theorem 5.5] the proof is complete.

THEOREM 4.4. Under the hypotheses of Theorem 4.3, suppose that moreover for $\alpha$ given by 4.4 one of the following holds

(i) $\alpha<0$ and there exists $\theta_{0} \in \Theta$ such that,

$$
\kappa\left(\theta_{0}\right)>0
$$

(ii) $\alpha \geq 0$ and for every $\theta \in \Theta$

$$
\kappa(\theta)>0
$$

Then operator $P$ given by 3.1 is asymptotically stable.

Proof. By Theorem 4.3 the operator $P$ admits an invariant measure. Using [9, Theorems 5.4 and 5.5] it is sufficient to show that for given $\epsilon>0$ there exists $\gamma>0$ such that for any two measures $\mu_{1}, \mu_{2} \in M_{1}$, there exist a Borel measurable set $A \subset X$ with $\operatorname{diam}_{\bar{\rho}_{\varphi}}(A)<\epsilon$ and an integer $n$ such that

$$
P^{n} \mu_{k}(A) \geq \gamma \quad \text { for } k=1,2
$$

By [9, Theorem 5.5], the set $L\left(M_{1}\right)$ is tight. Thus there exists a compact set $F \subset X$ such that

$$
\mu(F) \geq \frac{4}{5} \quad \text { for every } \mu \in L\left(M_{1}\right)
$$

Consider two cases: $\alpha<0$ and $\alpha \geq 0$. 
Case 1: $\alpha<0$. Fix $\epsilon_{1}>0$ and $i_{0} \in I$. There exist $\varepsilon>0$ such that $\varphi(\varepsilon)<\epsilon_{1}$. Choose $t_{*} \in R_{+}$such that

$$
\frac{L l_{q}}{\sigma \kappa\left(\theta_{0}\right)} e^{\alpha t_{*}} \operatorname{diam}_{\bar{\rho}}(F)<\frac{\epsilon}{2} .
$$

Define

$$
F_{Y}=\{x \in Y:(x, i) \in F \text { for some } i \in I\}
$$

Clearly $F_{Y}$ is a compact subset of $Y$.

Since $q: Y \times \Theta \rightarrow Y$ and $S_{i}: R_{+} \times Y \rightarrow Y, i \in I$, are continuous, there exists $\bar{t}>t_{*}$ such that

$$
\left\|q\left(S_{i}(t, x), \theta_{0}\right)-q\left(S_{i}\left(t_{*}, x\right), \theta_{0}\right)\right\|<\frac{\epsilon}{8}
$$

for every $i \in I, x \in F_{Y}, t \in\left[t_{*}, \bar{t}\right]$, where $\theta_{0}$ is given by condition 4.12). Now for $x \in F_{Y}$ we set

$$
\begin{aligned}
U(x)=\left\{z \in F_{Y}:\left\|q\left(S_{i}\left(t_{*}, z\right), \theta_{0}\right)-q\left(S_{i}\left(t_{*}, x\right), \theta_{0}\right)\right\|\right. & <\frac{\epsilon}{8} \\
& \text { for every } i \in I\} .
\end{aligned}
$$

By the compactness of $F_{Y}$, we choose $z_{1}, \ldots, z_{m_{0}}$, such that $F \subset G$, where

$$
G=\bigcup_{l=1}^{m_{0}}\left(U\left(z_{l}\right) \times I\right) .
$$

The set $G$ is an open subset of $X$. Let $\mu_{1}, \mu_{2} \in M_{1}$ be arbitrary. Set $\mu=$ $\left(\mu_{1}+\mu_{2}\right) / 2$. Since $L(\mu) \neq \emptyset$, there exist a subsequence $\left\{n_{k}\right\}$ of $\{n\}$ and a measure $\nu \in L(\mu)$ such that $P^{n_{k}} \mu \rightarrow \nu$ (weakly). Since $\nu(F) \geq \frac{4}{5}$, the Aleksandrov theorem implies

$$
\liminf _{k \rightarrow \infty} P^{n_{k}} \mu(G) \geq \nu(G) \geq \frac{4}{5} .
$$

It follows that there exists $n_{0} \in N$ such that

$$
P^{n_{0}} \mu(G)=\frac{P^{n_{0}} \mu_{1}(G)+P^{n_{0}} \mu_{2}(G)}{2} \geq \frac{3}{4} .
$$


Consequently

$$
P^{n_{0}} \mu_{k}(G) \geq \frac{1}{2} \quad \text { for } k=1,2 .
$$

From the definition of $G$ and 4.17 there exist $l_{1}, l_{2} \in\left\{1, \ldots, m_{0}\right\}$ and $i_{1}, i_{2}$ such that

$$
P^{n_{0}} \mu_{k}\left(v_{k}\right) \geq \frac{1}{2 m_{0} N} \quad \text { for } k=1,2,
$$

where

$$
v_{k}=U\left(z_{l_{k}}\right) \times\left\{i_{k}\right\} \quad \text { for } k=1,2 .
$$

From (4.4) we have

$$
\left\|S_{i_{0}}\left(t_{*}, z_{l_{1}}\right)-S_{i_{0}}\left(t_{*}, z_{l_{2}}\right)\right\| \leq \frac{L}{\sigma} e^{\alpha t_{*}}\left\|z_{l_{1}}-z_{l_{2}}\right\| .
$$

Set

$$
w_{1}=S_{i_{0}}\left(t_{*}, z_{l_{1}}\right) \quad \text { and } \quad w_{2}=S_{i_{0}}\left(t_{*}, z_{l_{2}}\right) .
$$

From condition (4.5) it follows that

$$
\left\|q\left(w_{1}, \theta_{0}\right)-q\left(w_{2}, \theta_{0}\right)\right\| \leq \frac{l_{q}}{\kappa\left(\theta_{0}\right)}\left\|w_{1}-w_{2}\right\| .
$$

By 4.20, 4.19) and 4.14 we obtain

$$
\left\|q\left(w_{1}, \theta_{0}\right)-q\left(w_{2}, \theta_{0}\right)\right\| \leq \frac{l_{q}}{\kappa\left(\theta_{0}\right)}\left\|w_{1}-w_{2}\right\| \leq \frac{L l_{q}}{\sigma \kappa\left(\theta_{0}\right)} e^{\alpha t_{*}}\left\|z_{l_{1}}-z_{l_{2}}\right\| \leq \frac{\epsilon}{2} .
$$

Define

$$
A=\left(B\left(q\left(w_{1}, \theta_{0}\right), \frac{\epsilon}{4}\right) \cup B\left(q\left(w_{2}, \theta_{0}\right), \frac{\epsilon}{4}\right)\right) \times\left\{i_{0}\right\} .
$$

Observe that $\operatorname{diam}_{\bar{\rho}_{\varphi}}(A)<\epsilon_{1}$. For $x \in U\left(z_{l_{1}}\right)$ and $t \in\left[t_{*}, \bar{t}\right]$ using 4.15) and 4.16) we have

$$
\begin{aligned}
& \left\|q\left(S_{i_{0}}(t, x), \theta_{0}\right)-q\left(w_{1}, \theta_{0}\right)\right\| \leq\left\|q\left(S_{i_{0}}(t, x), \theta_{0}\right)-q\left(S_{i_{0}}\left(t_{*}, x\right), \theta_{0}\right)\right\| \\
& \quad+\left\|q\left(S_{i_{0}}\left(t_{*}, x\right), \theta_{0}\right)-q\left(S_{i_{0}}\left(t_{*}, z_{l_{1}}\right), \theta_{0}\right)\right\| \leq \frac{\epsilon}{8}+\frac{\epsilon}{8}=\frac{\epsilon}{4} .
\end{aligned}
$$


This gives

$$
\left(q\left(S_{i_{0}}(t, x), \theta_{0}\right), i_{0}\right) \in A \quad \text { for } x \in U\left(z_{l_{1}}\right), t \in\left[t_{*}, \bar{t}\right] .
$$

Similarly,

$$
\left(q\left(S_{i_{0}}(t, x), \theta_{0}\right), i_{0}\right) \in A \quad \text { for } x \in U\left(z_{l_{2}}\right), t \in\left[t_{*}, \bar{t}\right]
$$

Using (4.21) and 4.18), for $i=1,2$ we have

$$
\begin{aligned}
& P^{n_{0}+1} \mu_{i}(A)= \sum_{s=1}^{N} \int_{X} \int_{0}^{\infty} \int_{\Theta} 1_{A}\left(q\left(S_{s}(t, x), \theta\right), s\right) e^{-L(t, x, k)} \\
& \times \lambda\left(S_{k}(t, x)\right) p_{k s}(x) d \kappa(\theta) d t P^{n_{0}} \mu_{i}(d x, d k) \\
& \geq \int_{v_{k}} \int_{t_{*}}^{\bar{t}} \int_{\Theta} 1_{A}\left(q\left(S_{i_{0}}(t, x), \theta_{0}\right), i_{0}\right) e^{-L(t, x, k)} \\
& \geq\left.\int_{v_{k}} \int_{t_{*}}^{\bar{t}} \kappa\left(\theta_{0}\right) e^{-L(t, x, k)} \lambda\left(S_{k}(t, x)\right) p_{k i_{0}}(x) d t P^{n_{0}} \mu_{i}(d x, d k)\right) p_{k i_{0}}(x) d \kappa(\theta) d t P^{n_{0}} \mu_{i}(d x, d k) \\
& \geq \int_{v_{k}} \int_{t_{*}}^{\bar{t}} \kappa\left(\theta_{0}\right) e^{-\bar{\lambda} t} \underline{\lambda} \sigma d t P^{n_{0}} \mu_{i}(d x, d k) \\
&=\kappa\left(\theta_{0}\right) \frac{\sigma \underline{\lambda}}{\bar{\lambda}}\left(e^{-\bar{\lambda} t_{*}}-e^{-\bar{\lambda} \bar{t}}\right) \frac{1}{2 m_{0} N}=\gamma .
\end{aligned}
$$

For $i=1,2$ the constant $\gamma$ does not depend on $\mu_{i}$. The proof of the first case is completed.

CASE 2: $\alpha \geq 0$. Let $\epsilon_{1}>0$ and $i_{0} \in I$ be fixed. There exist $\varepsilon>0$, such that $\varphi(\varepsilon)<\epsilon_{1}$. We introduce some further notations

$$
\begin{gathered}
\left.\mathbf{q}_{\theta}(x)=q\left(q\left(q\left(x, \theta_{1}\right), \ldots\right), \theta_{n-1}\right), \theta_{n}\right), \\
\left(\mathbf{q}_{\theta} \circ S_{i}\right)(\tau, x)=q\left(S_{i_{n}}\left(\tau_{n}, q\left(S_{i_{n-1}}\left(\tau_{n-1}, \ldots, S_{i_{1}}\left(\tau_{1}, x\right)\right), \theta_{n-1}\right), \theta_{n}\right),\right. \\
d \tau=d \tau_{1} \cdots d \tau_{n}, \\
d \theta=d \theta_{1} \cdots d \theta_{n} .
\end{gathered}
$$


Next, for $n \geq 2$, consider functions: $P_{n}: Y \times I^{n+1} \times R_{+}^{n-1} \times \Theta^{n-1} \rightarrow[0,1]$ given by

$$
\begin{aligned}
P_{n}\left(x, k, i_{1}, \ldots,\right. & \left.i_{n-1}, i_{n}, \tau_{1}, \ldots, \tau_{n-1}, \theta_{1}, \ldots, \theta_{n-1}\right) \\
& =p_{k i_{1}}(x) p_{i_{1} i_{2}}\left(q\left(S_{i_{1}}\left(\tau_{1}, x\right), \theta_{1}\right)\right) \cdots p_{i_{n-1} i_{n}}\left(\left(\mathbf{q}_{\theta} \circ \mathbf{S}_{\mathbf{i}}\right)(\tau, x)\right),
\end{aligned}
$$

$\Lambda_{n}: Y \times I^{n} \times R_{+}^{n} \times \Theta^{n-1} \rightarrow(0, \infty)$, where

$$
\begin{aligned}
& \Lambda_{n}\left(x, k, i_{1}, \ldots, i_{n-1}, \tau_{1}, \ldots, \tau_{n-1}, \tau_{n}, \theta_{1}, \ldots, \theta_{n-1}\right) \\
& =\lambda\left(S_{k}\left(\tau_{1}, x\right)\right) \lambda\left(S_{i_{1}}\left(\tau_{2}, q\left(S_{i_{1}}\left(\tau_{1}, x\right), \theta_{1}\right)\right) \cdots \lambda\left(S_{i_{n-1}}\left(\tau_{n},\left(\mathbf{q}_{\theta} \circ \mathbf{S}_{\mathbf{i}}\right)(\tau, x)\right)\right),\right.
\end{aligned}
$$

$\Sigma_{n}: Y \times I^{n} \times R_{+}^{n} \times \Theta^{n-1}$, where

$$
\begin{aligned}
\Sigma_{n}\left(x, k, i_{1}, \ldots, i_{n-1}, \tau_{1}, \ldots, \tau_{n}, \theta_{1}, \ldots, \theta_{n-1}\right) & \\
& =e^{-L\left(\tau_{1}, x, k\right)} e^{-L\left(\tau_{2}, q\left(S_{i_{1}}\left(\tau_{1}, x\right), \theta_{1}\right), i_{1}\right)} \cdots e^{-L\left(\tau_{n},\left(\mathbf{q}_{\theta} \circ \mathbf{S}_{\mathbf{i}}\right)(\tau, x), i_{n-1}\right)},
\end{aligned}
$$

where $\theta=\left(\theta_{1}, \ldots, \theta_{n-1}\right), \tau=\left(\tau_{1}, \ldots, \tau_{n-1}\right), \mathbf{i}=\left(i_{1}, \ldots, i_{n-1}\right)$. From assumption 4.7) we have that $l_{q}<1$. Let $n \in N$ be such that

$$
l_{q}^{n} \operatorname{diam}_{\bar{\rho}}(F)<\frac{\epsilon}{2} .
$$

By continuity and compactness there exists $\delta>0$ such that

$$
\left\|\left(\mathbf{q}_{\theta} \circ S_{i}\right)(\tau, x)-\mathbf{q}_{\theta}(x)\right\| \leq \frac{\epsilon}{8}
$$

for every $i \in I, \theta \in \Theta^{n}, \tau \in[0, \delta]^{n}$ and $x \in F_{Y}$, where

$$
F_{Y}=\{x \in Y:(x, i) \in F \text { for some } i \in I\} .
$$

Given $x \in Y$ define

$$
U(x)=\left\{z \in F_{Y}:\left\|\mathbf{q}_{\theta}(x)-\mathbf{q}_{\theta}(z)\right\|<\frac{\epsilon}{8} \text { for } \theta \in \Theta^{n}\right\} .
$$

Let $z_{1}, \ldots, z_{m_{0}} \in F_{Y}$ be such that $F \subset G$, where

$$
G=\bigcup_{l=1}^{m_{0}}\left(U\left(z_{l}\right) \times I\right) .
$$


Let $\mu_{1}, \mu_{2} \in M_{1}$. Set $\mu=\left(\mu_{1}+\mu_{2}\right) / 2$. Since $L(\mu) \neq \emptyset$, there exist a subsequence $\left\{n_{k}\right\}$ of $\{n\}$ and a measure $\nu \in L(\mu)$ such that $P^{n_{k}} \mu \rightarrow \nu$. There exist $n_{0} \in N, l_{1}, l_{2} \in\left\{1, \ldots, m_{0}\right\}$ and $i_{1}, i_{2}$ such that

$$
P^{n_{0}} \mu_{g}\left(v_{g}\right) \geq \frac{1}{2 m_{0} N} \quad \text { for } g=1,2,
$$

where

$$
v_{g}=U\left(z_{l_{g}}\right) \times\left\{i_{g}\right\} \quad \text { for } g=1,2 .
$$

From 4.5, there exist $\theta_{0,0}$ such that

$$
\left\|q\left(z_{l_{1}}, \theta_{0,0}\right)-q\left(z_{l_{2}}, \theta_{0,0}\right)\right\| \leq l_{q}\left\|z_{l_{1}}-z_{l_{2}}\right\| .
$$

For $q\left(z_{l_{1}}, \theta_{0,0}\right)$ and $q\left(z_{l_{2}}, \theta_{0,0}\right)$ we choose $\theta_{0,1}$ such that

$$
\left\|q\left(q\left(z_{l_{1}}, \theta_{0,0}\right), \theta_{0,1}\right)-q\left(q\left(z_{l_{2}}, \theta_{0,0}\right), \theta_{0,1}\right)\right\| \leq l_{q}\left\|q\left(z_{l_{1}}, \theta_{0,0}\right)-q\left(z_{l_{2}}, \theta_{0,0}\right)\right\|
$$

and so on. Thus there exists $\theta_{\mathbf{0}}=\left(\theta_{0,0}, \ldots, \theta_{0, n-1}\right) \in \Theta^{n}$ such that

$$
\left\|\mathbf{q}_{\theta_{\mathbf{0}}}\left(z_{l_{1}}\right)-\mathbf{q}_{\theta_{\mathbf{0}}}\left(z_{l_{2}}\right)\right\| \leq l_{q}^{n}\left\|z_{l_{1}}-z_{l_{2}}\right\| .
$$

Define

$$
A=\left(B\left(\mathbf{q}_{\theta_{\mathbf{0}}}\left(z_{l_{1}}\right), \frac{\epsilon}{4}\right) \cup B\left(\mathbf{q}_{\theta_{\mathbf{0}}}\left(z_{l_{2}}\right), \frac{\epsilon}{4}\right)\right) \times\left\{i_{0}\right\} .
$$

From 4.26) and 4.22 it follows that $\operatorname{diam}_{\bar{\rho}_{\varphi}}(A)<\epsilon_{1}$. For $x \in U\left(z_{l_{g}}\right), g=$ $1,2, \mathbf{i} \in I^{n}$ and $\tau \in[0, \delta]^{n}$ from 4.23 and 4.24 we have

$$
\begin{aligned}
\left\|\left(\mathbf{q}_{\theta_{\mathbf{0}}} \circ \mathbf{S}_{\mathbf{i}}\right)(\tau, x)-\mathbf{q}_{\theta_{\mathbf{0}}}\left(z_{l_{k}}\right)\right\| \leq \|\left(\mathbf{q}_{\theta_{\mathbf{0}}} \circ \mathbf{S}_{\mathbf{i}}\right)(\tau, x) & -\mathbf{q}_{\theta_{\mathbf{0}}}(x) \| \\
& +\left\|\mathbf{q}_{\theta_{\mathbf{0}}}(x)-\mathbf{q}_{\theta_{\mathbf{0}}}\left(z_{l_{k}}\right)\right\| \leq \frac{\epsilon}{4} .
\end{aligned}
$$

This gives $\left(\left(\mathbf{q}_{\theta_{\mathbf{0}}} \circ \mathbf{S}_{\mathbf{i}}\right)(\tau, x), i_{0}\right) \in A$ for $x \in U\left(z_{l_{g}}\right), \mathbf{i} \in I^{n}, g=1,2$ and $\tau \in$ $[0, \delta]^{n}$. Combining this with 4.25 and using

$$
\begin{aligned}
& P^{n_{0}+n} \mu_{g}(A)= \sum_{\mathbf{i}=\left(i_{1}, \ldots, i_{n}\right) \in I^{n}} \int_{X} \int_{R_{+}{ }^{n}} \int_{\boldsymbol{\Theta}=\left(\theta_{1}, \ldots, \theta_{n}\right) \in \Theta^{n}} \mathbb{1}_{A}\left(\left(\mathbf{q}_{\theta} \circ \mathbf{S}_{\mathbf{i}}\right)(\tau, x), i_{n}\right) \\
& \times P_{n}\left(x, k, \mathbf{i}, \tau_{1}, \ldots, \tau_{n-1}, \theta_{1}, \ldots, \theta_{n-1}\right) \Lambda_{n}\left(x, k, i_{1}, \ldots, i_{n-1}, \tau, \theta_{1}, \ldots, \theta_{n-1}\right) \\
& \times \Sigma_{n}\left(x, k, i_{1}, \ldots, i_{n-1}, \tau_{1}, \ldots, \tau_{n-1}\right) d \tau \kappa(\theta) P^{n_{0}} \mu_{g}(d x, d k),
\end{aligned}
$$


we obtain

$$
\begin{aligned}
P^{n_{0}+n} \mu_{g}(A) \geq \sigma^{n} \underline{\lambda}^{n} \int_{v_{g}} \int_{[0, \delta]^{n}} \mathbb{1}_{A}\left(\left(\mathbf{q}_{\theta_{\mathbf{0}}} \circ \mathbf{S}_{\mathbf{i}_{\mathbf{o}}}\right)(\tau, x), i_{0}\right) \\
\quad \times e^{-\bar{\lambda}\left(\tau_{1}+\ldots+\tau_{n}\right)} d \tau \kappa(\theta) P^{n_{0}} \mu_{g}(d x, d k) \\
\geq(\sigma \underline{\underline{\lambda}})^{n} \int_{[0, \delta]^{n}} e^{-\bar{\lambda}\left(\tau_{1}+\ldots+\tau_{n}\right)} d \tau P^{n_{0}} \mu_{g}\left(v_{g}\right) \\
\geq\left(\frac{\sigma \underline{\lambda}}{\bar{\lambda}}\right)^{n} \frac{\left(1-e^{-\bar{\lambda} \sigma}\right)^{n}}{2 m_{0} N}=\gamma
\end{aligned}
$$

For $g=1,2$ the constant $\gamma$ does not depend on $\mu_{g}$. The proof of the second case is completed.

EXAMPLE 4.5. In paper [7] there was introduced a model of gene expression. This model involves three classes of processes: allele activation/inactivation, mRNA transcription/decay, and protein translation/decay process. It is assumed that, due to binding or dissociation of protein molecules, each of gene's alleles may be transformed, independently of the remaining ones, into an active state or into an inactive state.

In this example we consider similar model like this in [7]. In $R^{2}$ with norm $\|x\|=\left|x_{1}\right|+\left|x_{2}\right|$, we consider Lipschitz function $\lambda: R^{2} \rightarrow(0, \infty)$ such that

$$
0<\underline{\lambda}<\bar{\lambda}<\infty \text { and } \bar{\lambda}-1<\underline{\lambda} \text {. }
$$

Set $\Theta=\{1\}$, let $q: R^{2} \times\{1\} \rightarrow R^{2}$ be such that

$$
\|q(x, 1)-q(y, 1)\| \leq l_{q}\|x-y\| \quad \text { for } x, y \in R^{2} .
$$

Further, let

$$
p_{00}(x)=p_{01}(x)=p_{10}(x)=p_{11}(x)=\frac{1}{2} \quad \text { for all } x \in R^{2} .
$$

We consider

$$
\begin{aligned}
& \frac{d x_{1}^{i}}{d t}=i-x_{1}^{i}, \\
& \frac{d x_{2}^{i}}{d t}=r\left(x_{1}^{i}-x_{2}^{i}\right),
\end{aligned}
$$


where $i \in\{0,1\}$ and $r \in R$. The solutions are of the form

$$
\begin{aligned}
& x_{1}^{i}(t)=i+\left(x_{1}-i\right) e^{-t}, \\
& x_{2}^{i}(t)=\left\{\begin{array}{l}
x_{2} e^{-r t}+\frac{r}{r-1}\left(x_{1}-i\right)\left(e^{-t}-e^{-r t}\right)+i\left(1-e^{-r t}\right), \quad \text { if } r \neq 1, \\
x_{2} e^{-t}+\left(x_{1}-i\right) e^{-t} t+i\left(1-e^{-t}\right), \quad \text { if } r=1 .
\end{array}\right.
\end{aligned}
$$

We only consider the case when $r>1$. For $i=0$ we set

$$
S_{0}(t, x)=\left(x_{1} e^{-t}, x_{2} e^{-r t}+\frac{r}{r-1} x_{1}\left(e^{-t}-e^{-r t}\right)\right),
$$

and for $i=1$

$$
S_{1}(t, x)=\left(1+\left(x_{1}-1\right) e^{-t}, x_{2} e^{-r t}+\frac{r}{r-1}\left(x_{1}-1\right)\left(e^{-t}-e^{-r t}\right)+\left(1-e^{-r t}\right)\right),
$$

where $t \geq 0$ and $x=\left(x_{1}, x_{2}\right)$. We calculate

$$
\begin{aligned}
\left\|S_{0}(t, x)-S_{0}(t, y)\right\| \leq & e^{-t}\left|x_{1}-y_{1}\right|+e^{-r t}\left|x_{2}-y_{2}\right| \\
& +\frac{r}{r-1}\left(e^{-t}-e^{-r t}\right)\left|x_{1}-y_{1}\right| \\
= & \left|x_{1}-y_{1}\right| \frac{(2 r-1) e^{-t}-r e^{-r t}}{r-1}+e^{-r t}\left|x_{2}-y_{2}\right| \\
\leq & \left|x_{1}-y_{1}\right| \frac{(2 r-1) e^{-t}}{r-1}+e^{-r t}\left|x_{2}-y_{2}\right| \\
= & \left|x_{1}-y_{1}\right| \frac{(2 r-1) e^{-t}}{r-1}+\frac{(2 r-1) e^{-t}}{r-1}\left|x_{2}-y_{2}\right| \\
& =\frac{(2 r-1) e^{-t}}{r-1}\|x-y\|
\end{aligned}
$$

and

$$
\begin{aligned}
\left\|S_{1}(t, x)-S_{1}(t, y)\right\| \leq & e^{-t}\left|x_{1}-y_{1}\right|+e^{-r t}\left|x_{2}-y_{2}\right| \\
& +\frac{r}{r-1}\left(e^{-t}-e^{-r t}\right)\left|x_{1}-y_{1}\right| \\
\leq & \frac{(2 r-1) e^{-t}}{r-1}\|x-y\| .
\end{aligned}
$$

We check when the operator $P$ given by (3.1) for the considered functions $\lambda, q, S_{0}, S_{1}, p_{k s}$ is asymptotically stable. It is easy to check that conditions 
4.1)-4.6 are satisfied. Moreover, if $l_{q}<(r-1) /(2 r-1)$ then 4.7) holds, because we have $L=(2 r-1) /(r-1), \alpha=-1$, and

$$
L l_{q} \bar{\lambda}+\alpha=\frac{(2 r-1)}{r-1} l_{q} \bar{\lambda}-1<\bar{\lambda}-1<\underline{\lambda}
$$

\section{References}

[1] Davis M.H.A., Markov Models and Optimization, Chapman and Hall, London, 1993.

[2] Diekmann O., Heijmans H.J., Thieme H.R., On the stability of the cells size distribution, J. Math. Biol. 19 (1984), 227-248.

[3] Horbacz K., Asymptotic stability of a system of randomly connected transformations on Polish spaces, Ann. Polon. Math. 76 (2001), 197-211.

[4] Horbacz K., Invariant measures for random dynamical systems, Dissertationes Math. 451 (2008), 68 pp.

[5] Kazak J., Piecewise-deterministic Markov processes, Annales Polonici Mathematici 109 (2013), 279-296.

[6] Lasota A., Yorke J.A., Lower Bound technique for Markov operators and iterated function systems, Random Comput. Dynam. 2 (1994), 41-77.

[7] Lipniacki T., Paszek P., Marciniak-Czochra A., Brasier A.R., Kimel M., Transcriptional stochasticity in gene expression, J. Theor. Biol. 238 (2006), 348-367.

[8] Snyder D., Random Point Processes, Wiley, New York, 1975.

[9] Szarek T., Invariant measures for nonexpansive Markov operators on Polish spaces, Dissertationes Math. 415 (2003), 62 pp.

Institute of Mathematics

University of Silesia

BANKOWA 14

40-007 Katowice

PolAnd

e-mail: jkubieniec@us.edu.pl 